\title{
Teor de mercúrio em peixes do Nordeste brasileiro: uma revisão integrativa
}

Essa pesquisa consiste em uma revisão integrativa visando discutir sobre a concentração de mercúrio presente em peixes da região Nordeste do Brasil e identifica suas implicações na saúde. A revisão foi composta por seis etapas distintas: 1) elaboração a pergunta norteadora; 2) busca ou amostragem na literatura; 3) coleta de dados; 4) análise crítica dos estudos incluídos; 5) discussão dos resultados e 6) apresentação da revisão integrativa. A leitura dos títulos e resumos foi realizada de forma independente por dois revisores nas bases de dados Scientific Electronic Library Online (SciELO), Portal de Periódicos da Coordenação de Aperfeiçoamento de Pessoal de Nível Superior (CAPES), ScienceDirect e buscador Google Acadêmico. A coleta de dados foi realizada entre Abril e Agosto de 2018, incluindo os termos mercúrio, peixe, concentração, Nordeste e Brasil para a busca. Selecionou-se seis estudos observacionais. Os maiores níveis de Mercúrio (Hg) foram encontrados nas espécies S. cavalla $(444,8 \pm 519,9 \mathrm{ng} / \mathrm{g}-1)$, R. porosus $(509 \mathrm{ng} / \mathrm{g}-1)$ e G. cirratum $(500 \pm 330 \mathrm{ng} / \mathrm{g}-1)$. Em quatro estudos incluídos nesta revisão verificou-se a concentração de $\mathrm{Hg}$ em peixes e sua relação com o comprimento e o peso. Concluiu-se que os peixes da região nordeste do Brasil apresentam, em média, concentrações totais de mercúrio abaixo do limite considerado seguro para consumo estabelecido pelas normas do Mercosul.

Palavras-chave: Mercúrio; Peixes; Contaminação de Alimentos.

\section{Content of mercury in fishes from Brazilian Northeast: a integrative review}

\begin{abstract}
This research consists of an integrative review aiming to discuss mercury concentration present in fishes from the Northeast region of Brazil and identify its implication for health. The review was composed of six distinct stages: 1) elaboration of the guiding question; 2) searching or sampling in the literature; 3) data collection; 4) critical analysis of the included studies; 5) discussion of results and 6) presentation of the integrative review. Titles and abstracts were read independently by two reviewers in the database of Scientific Electronic Library Online (SciELO), Coordination for the Improvement of Higher Education Personnel (CAPES), ScienceDirect and Google Scholar. Data collection was carried out between April and August of 2018 by including the terms: mercury, fish, concentration, Northeast and Brazil for the search. Six observational studies were selected. The greatest levels of Mercury ( $\mathrm{Hg}$ ) were found in the species $\mathrm{S}$. cavalla (444,8 $\pm 519,9$ $\mathrm{ng} / \mathrm{g}-1)$, R. porosus (509 $\mathrm{ng} / \mathrm{g}-1)$ and G. cirratum $(500 \pm 330 \mathrm{ng} / \mathrm{g}-1)$. In four studies included in this review were verified Hg concentration in fishes and its relation with length and weight. It was concluded that fish from Brazilian Northeast presented, on average, a total concentration of mercury below the limit considered safe for consumption, established by Southern Common Market (Mercosur) rules.
\end{abstract}

Keywords: Mercury; Fish; Food Contamination.

Topic: Oceanografia Física, Química, Geológica, Biológica

Reviewed anonymously in the process of blind peer.
Received: 02/01/2021

Approved: 27/01/2021
Sarah Lopes Rodrigues (D)

Universidade Estadual do Ceará, Brasil

http://lattes.cnpq.br/3731768511750268

http://orcid.org/0000-0003-2352-2939

sarahlopesnutri@gmail.com

Renata Carmo de Assis (iD

Universidade Estadual do Ceará, Brasil

http://lattes.cnpq.br/5668625762438217

http://orcid.org/0000-0003-2258-3844

renata.carmo.assis@gmail.com

Roberta Freitas Celedônio (iD)

Universidade Estadual do Ceará, Brasil

http://lattes.cnpq.br/6710890976135835

http://orcid.org/0000-0003-0787-8070

robertta.celedonio@aluno.uece.br

\author{
Ashley Brito Valentim (iD \\ Universidade Estadual do Ceará, Brasil \\ http://lattes.cnpq.br/3942230637437049 \\ http://orcid.org/0000-0001-9485-715X \\ ashley.valentim@aluno.uece.br \\ Carla Soraya Costa Maia (iD \\ Universidade Estadual do Ceará, Brasil \\ http://lattes.cnpq.br/2879750916488596 \\ http://orcid.org/0000-0003-1535-6686 \\ carla.maia@uece.br
}

Referencing this:

RODRIGUES, S. L.; ASSIS, R. C.; CELEDONIO, R. F.; VALENTIM, A. B.; MAIA, C. S. C.. Teor de mercúrio em peixes do Nordeste brasileiro: uma revisão integrativa. Revista Ibero Americana de Ciências Ambientais, v.12, n.1, p.223-231, 2021. DOI:

http://doi.org/10.6008/CBPC2179-6858.2021.001.0019 


\section{INTRODUÇÃO}

O mercúrio $(\mathrm{Hg})$ é um elemento natural de ampla distribuição que está presente na superfície da terra, na água, na atmosfera e também em seres vivos. Ocorrendo em concentrações variadas e associado a outros elementos. O Hg é emitido para a superfície atmosférica por meio de fontes naturais como: Erupção vulcânica, evaporação em solo e evaporação em água da superfície dos oceanos e fontes artificiais como: as indústrias, extração de mercúrio e queima de combustíveis fósseis (MICARONI et al., 2000; ANVISA, 2013).

Esse metal pode ser encontrado na forma de espécies orgânicas ou inorgânicas. Na forma inorgânica pode se apresentar em estados de oxidação diferentes, como exemplo tem-se $\mathrm{o}, \mathrm{Hg}_{2}{ }^{2+}, \mathrm{Hg}^{2+}$ e $\mathrm{Hg}$ elementar $\left(\mathrm{Hg}^{0}\right)$ que pode ser encontrado, principalmente, na forma de gás ou na forma líquida. Na forma orgânica o metilmercúrio (MeHg) é o mais comum e o mais importante por sua considerável toxicidade. A forma do Hg elementar $\left(\mathrm{Hg}^{0}\right)$ pode ser oxidado na presença de ozônio e ser carreado para a superfície pela precipitação seca e úmida. Por meio de um processo chamado de biometilação, o $\mathrm{Hg}^{0}$ pode ser convertido em metilmercúrio, esse processo é realizado por bactérias que tem ação intensa favorecida no ambiente aquático, por condições ótimas de temperatura e pH, e também elevada quantidade de matéria orgânica. Uma vez metilado, o Hg aumenta sua concentração ao longo do tempo de exposição ao organismo, processo chamado de bioacumulação (LACERDA et al., 2008).

Os níveis de exposição ao Hg variam de acordo com áreas impactadas. Áreas que ocorrem atividades garimpeiras de ouro, em que o mercúrio é utilizado na pré-concentração e extração do ouro, processo chamado de amalgamação. A atividade industrial também influencia na exposição ao $\mathrm{Hg}$, por exemplo, por meio da emissão de gases de efeito estufa e de metais, como o mercúrio, para a atmosfera. Essa emissão ocorre principalmente para geração de energia através da queima de combustíveis fósseis. A poluição das águas de peixes contaminados por mercúrio está associada a esses fatores (KASPER et al., 2007).

O mercúrio é introduzido a várias espécies via cadeia alimentar, incluindo plâncton, peixes herbívoros e carnívoros. Tais espécies contaminadas apresentam alteração nos órgãos e tecidos, podendo ocorrer também alterações neurológicas e comportamentais. Destaca-se então o peixe como fonte de contaminação por Hg para o ser humano, ressaltando que o grau de exposição ao mercúrio depende principalmente da frequência da inclusão de pescado na alimentação. No entanto, alguns fatores como a idade, o ciclo de vida, peso e sua posição na cadeia trófica influenciam a acumulação de mercúrio em peixes (MICARONI et al., 2000; BRABO et al., 1999, BURGER et al., 2003).

No Nordeste Brasileiro, a pesca é uma das principais atividades econômicas, constituindo-se uma essencial fonte de alimento para a população. Assim estudos buscam avaliar os níveis de exposição humana ao mercúrio pelo consumo e analisar pescados comercializados em determinadas regiões do país, incluindose a Região Nordeste, na qual é marcada pelo seu litoral e pela variedade de peixes comercializados (LACERDA et al., 2007; OLIVEIRA et al., 2016; TINÔCO et al., 2010).

Há grande importância de dados de concentração de Hg no consumo de peixes por se tratar de metal tóxico, a exposição excessiva ao metilmercúrio pode acarretar complicações clínicas como alterações no 
sistema nervoso central e periférico, pois o $\mathrm{MeHg}$, nos mamíferos, acumula-se preferencialmente nestes sistemas devido à sua afinidade com aminoácidos com enxofre presentes. Pode atingir a visão e audição, ocasiona também a falta de coordenação dos movimentos musculares levando a paralisia por causa da disfunção neural. A sintomatologia pode ser mais ainda mais grave em crianças (CARDOSO et al., 2009; SILVEIRA et al., 2004).

Diante do que foi exposto, fica claro a importância de se integrar as informações a respeito de dados de concentração de mercúrio em peixes no Nordeste brasileiro e identificar suas implicações na saúde, por meio de uma revisão integrativa que possa contribuir para fomentar ações e políticas de alimentação e nutrição nesta área.

\section{METODOLOGIA}

O presente estudo trata-se de uma revisão integrativa seguindo as etapas de Mendes et al. (2008), que é composta por seis etapas distintas: 1) elaboração a pergunta norteadora; 2) busca ou amostragem na literatura; 3) coleta de dados; 4) análise crítica dos estudos incluídos; 5) discussão dos resultados e 6) apresentação da revisão integrativa.

O estudo iniciou com a seguinte pergunta norteadora: 'quais os teores de mercúrio nos peixes da região nordeste do Brasil e suas implicações na saúde humana?' A busca ocorreu nas seguintes bases de dados: Scientific Electronic Library Online (SciELO), Portal de Periódicos da Coordenação de Aperfeiçoamento de Pessoal de Nível Superior (CAPES), ScienceDirect e buscador Google Acadêmico.

A estratégia de pesquisa incluiu os seguintes termos nas línguas portuguesa: mercúrio, peixe, concentração, Nordeste, Brasil e inglesa: mercury, fish, concentration, Northeast, Brazil. A coleta de dados foi realizada entre Abril e Agosto de 2018.

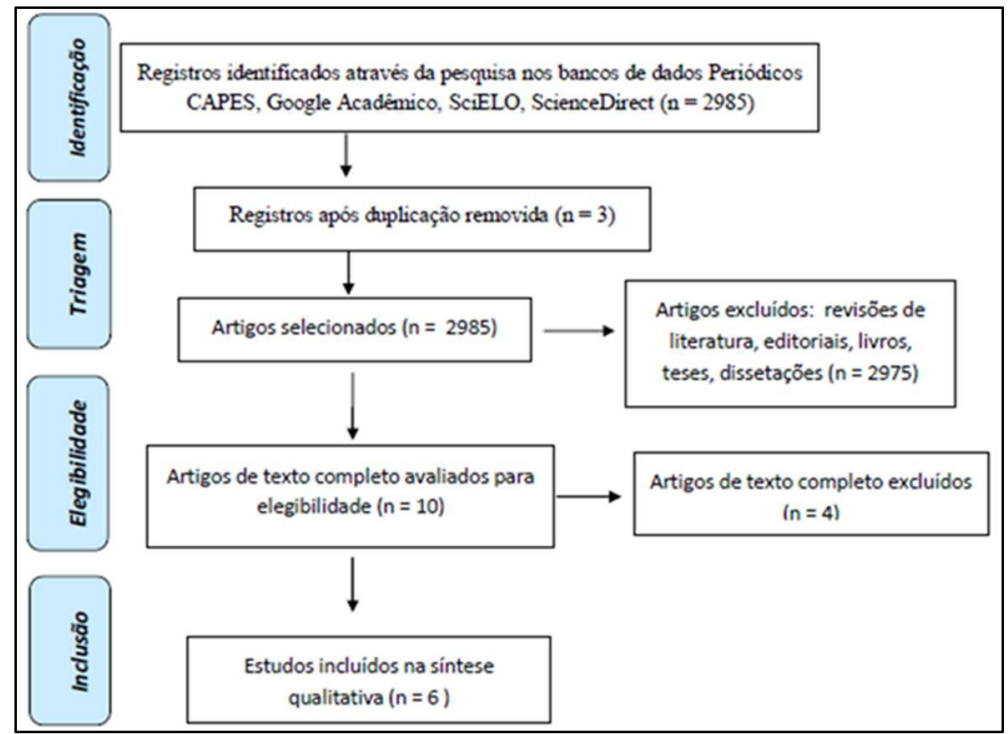

Figura 1: Fluxograma da seleção de estudos sobre mercúrio em peixes do Nordeste do Brasil.

Os critérios de inclusão foram artigos originais experimentais, revisões e meta-análises publicados a partir de janeiro de 2008 a dezembro de 2018, estudos realizados em peixes da região Nordeste do Brasil, publicados em português ou inglês. Foram excluídos livros, teses, dissertações e monografias. Estudos 
encontrados em mais de uma base de dados foram considerados apenas uma vez. A leitura dos títulos e resumos foi realizada de forma independente por dois revisores e então selecionados os artigos para a revisão. Os estágios de seleção dos artigos estão descritos na figura 1.

Foi medido coeficiente Kappa para avaliar a seleção, A classificação da taxa de concordância, segundo Landis et al. (1977), considera valores de intervalo $<0-1,00$, sendo $<0=$ sem concordância, $0-0,19=$ concordância leve, $0,20-0,39=$ razoável acordo, $0,40-0,59=$ concordância moderada, $0,60-0,79=$ concordância substancial e 0,80-1,00 = concordância quase perfeita.

\section{DISCUSSÃO TEÓRICA}

Com base nos critérios de elegibilidade, de 2.985 estudos encontrados na busca nas bases de dados, 10 artigos foram selecionados para leitura completa. Após a leitura, seis estudos observacionais foram selecionados para esta revisão. Com escore Kappa de 0,68 designando concordância substancial entre os revisores.

As características e os principais resultados dos estudos incluídos nesta revisão estão listados na Tabela 1. Desses estudos, o primeiro foi publicado em 2009 e o último em 2016. No que diz respeito à localização, quatro estudos foram realizados no Ceará, um em Pernambuco e um no Maranhão. Com relação ao número de espécies estudadas em cada artigo, esse número variou de um a 18 espécies. Em todos os seis estudos foram utilizadas amostras de músculo, um estudo utilizou também amostra de pele e fígado e um outro estudo utilizou amostras de pele, osso, guelras, intestino, rim, bexiga, fígado e coração. Quanto ao método para quantificar a concentração de $\mathrm{Hg}$ os seis estudos utilizaram espectrometria de absorção atômica com geração de vapor a frio (CV-AAS).

A presente revisão buscou analisar a concentração de mercúrio presente em peixes da região Nordeste do Brasil e identificar possíveis riscos de contaminação pelo consumo desses peixes. Segundo Brasil (2013) os limites estabelecidos pela legislação vigente para o consumo seguro de peixes são de $0,50 \mathrm{mgHg} / \mathrm{kg}$ ${ }^{1}$ para peixes não predadores e produtos de pesca, e de $1,00 \mathrm{mgHg} / \mathrm{kg}^{-1}$ para peixes predadores. Considerando-se então, níveis acima do estabelecido como potencial risco de contaminação.

\section{Concentração de mercúrio em peixes relacionada à posição na cadeia trófica}

O processo chamado biomagnificação consiste na maior concentração de mercúrio em níveis mais elevados da cadeia trófica. É observado que as maiores concentrações de Hg estão presentes em tecido muscular de peixes carnívoros e piscívoros, pois se encontram em níveis mais elevados da cadeia alimentar aquática. Nesses animais, a quantidade de mercúrio encontrada no organismo de peixes pode variar no tipo de tecido. O que mais apresenta relação com o armazenamento de $\mathrm{Hg}$ recente no organismo é o fígado, enquanto que, em outros tecidos estudados, músculo e pele, a concentração está relacionada principalmente à acumulação do metal ao longo do tempo (BURGER et al., 2003; COSTA et al., 2009a). 
Tabela 1: Principais características dos estudos incluídos na revisão sistemática.

\begin{tabular}{|c|c|c|c|c|c|}
\hline Autor, ano & $\begin{array}{l}\text { Número } \\
\text { espécies }\end{array}$ & Tipo de peixe & $\begin{array}{l}\text { Tipo de } \\
\text { amostra }\end{array}$ & Metodologia & Resultados encontrados \\
\hline $\begin{array}{l}\text { Costa et al. } \\
(2009)\end{array}$ & 2 & Piscívoro & $\begin{array}{l}\text { Músculo } \\
\text { Pele } \\
\text { Fígado }\end{array}$ & CV-AAS & $\begin{array}{l}\text { Hg total no } \\
\text { Músculo: } \\
\text { S. brasiliensis } 87 \quad \text { a } 497,0 \quad \mathrm{ng} / \mathrm{g}^{-1} \\
\text { S. cavalla } 36,0 \text { a } 1736,5 \mathrm{ng} / \mathrm{g}^{-1} \text {, } \\
\text { Fígado: } \\
\text { S. brasiliensis } 48,9-699,1 \mathrm{ng} / \mathrm{g}^{-1} \text {, } \\
\text { S. cavalla } 81,7-3901,9 \mathrm{ng} / \mathrm{g}^{-1} \text {, } \\
\text { Pele: } \\
\text { S. brasiliensis } 10,5-331,1 \mathrm{ng} / \mathrm{g}^{-1} \text {, } \\
\text { S. cavalla } 8,7-173,5 \mathrm{ng} / \mathrm{g}^{-1}\end{array}$ \\
\hline $\begin{array}{l}\text { Costa et al. } \\
(2009)\end{array}$ & 1 & Carnívoro & Músculo & CV-AAS & $\begin{array}{l}\text { As correlações entre as concentrações } \\
\text { logarítmicas totais de mercúrio }(\mathrm{Hg}-\mathrm{T}) \text { e as } \\
\text { variáveis biológicas Cumprimento Total e Peso } \\
\text { foram positivas. Média de } \mathrm{Hg}: 125,3 \mu \mathrm{g} / \mathrm{kg}^{-1}\end{array}$ \\
\hline $\begin{array}{l}\text { Costa et al. } \\
(2014)\end{array}$ & 13 & $\begin{array}{l}\text { Carnívoro } \\
\text { Onívoro } \\
\text { Detrítivoro }\end{array}$ & Músculo & CV-AAS & $\begin{array}{l}\text { A correlação entre a concentração de Hg e dados } \\
\text { morfométricos (peso e comprimento) foi } \\
\text { identificado apenas para algumas espécies } \\
\text { (Leporinus friderici, Serrasalmus rhombeus, } \\
\text { Eugerres brasilianus, Archosargus rhomboidalis e } \\
\text { Holocentrus adscensionis) }\end{array}$ \\
\hline $\begin{array}{l}\text { Oliveira et al. } \\
(2015)\end{array}$ & 1 & Herbívoro & $\begin{array}{l}\text { Músculo } \\
\text { Pele } \\
\text { Osso } \\
\text { Guelras } \\
\text { Intestino } \\
\text { Bexiga } \\
\text { Rim } \\
\text { Fígado } \\
\text { Coração }\end{array}$ & CV-AAS & 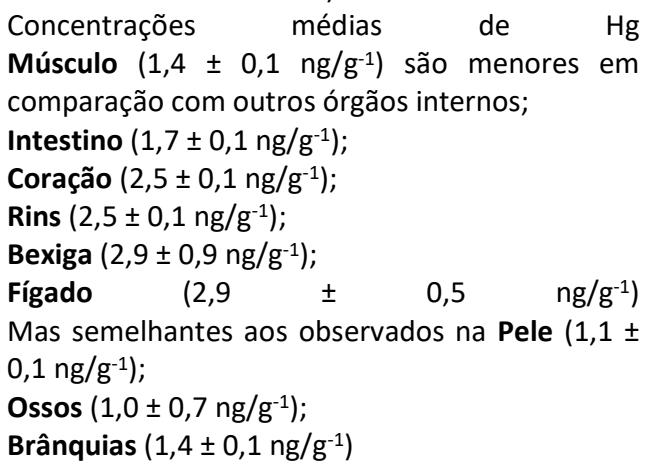 \\
\hline $\begin{array}{l}\text { Milhomem } \\
\text { Filho et al. } \\
(2016)\end{array}$ & 4 & $\begin{array}{l}\text { Piscívoro } \\
\text { Detritívoro } \\
\text { Onívoro } \\
\text { Herbívoro } \\
\text { Zooplanctófago }\end{array}$ & Músculo & CV-AAS & 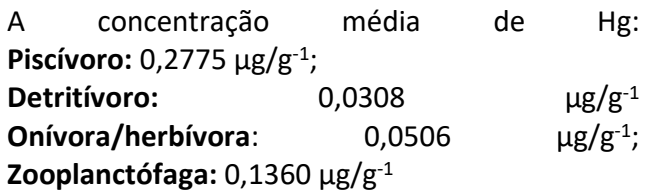 \\
\hline $\begin{array}{l}\text { Lacerda et al. } \\
(2016)\end{array}$ & 18 & $\begin{array}{l}\text { Carnívoro } \\
\text { Piscívoro } \\
\text { Planctívoro }\end{array}$ & Músculo & CV-AAS & $\begin{array}{l}\text { As maiores concentrações de Hg ocorreram em } \\
\text { espécies carnívoras e piscívoras grandes. } \\
\text { Principalmente, } \\
\text { S. cavalla com média de Hg } 352 \mathrm{ng} / \mathrm{g}^{-1} ; \mathrm{S} \text {. } \\
\text { brasiliensis com } \\
\begin{array}{l}\text { G. cirratum com } 500 \mathrm{ng} / \mathrm{g}^{-1} \text {; } \\
\text { R. porosus com } 509 \mathrm{ng} / \mathrm{g}^{-1}\end{array}\end{array}$ \\
\hline
\end{tabular}

CV-AAS: espectrometria de absorção atômica com geração de vapor a frio; Hg: mercúrio.

De acordo com Costa et al. (2009a) na região Nordeste do Brasil dentre as espécies de peixes mais comercializadas estão a Cavala e Serra sendo assim, uma via significativa de contaminação por mercúrio (Hg) para a população consumidora. Nesse estudo, determinaram o teor de $\mathrm{Hg}$ presente nas espécies de hábitos piscívoros Scomberomorus cavalla (Cavala) e Scomberomorus brasiliensis (Serra). O estudo mostrou menores concentrações na pele em ambas as espécies, com valores médios de 50,7 $\pm 49,4 \mathrm{ng} / \mathrm{g}^{-1} \mathrm{em} \mathrm{S}$. cavalla e 76,8 $\pm 96,9 \mathrm{ng} / \mathrm{g}^{-1} \mathrm{em} \mathrm{S}$. brasiliensis, enquanto que os maiores níveis de $\mathrm{Hg}$ foram encontrados no músculo de $S$. cavalla $\left(444,8 \pm 519,9 \mathrm{ng} / \mathrm{g}^{-1}\right)$ e de S. brasiliensis $\left(146,7 \pm 154,2 \mathrm{ng} / \mathrm{g}^{-1}\right)$. No peixe Cavala obteve-se um intervalo de 36,0 a $1736,5 \mathrm{ng} / \mathrm{g}^{-1}$ em indivíduos de 59 a $126 \mathrm{~cm}$, desse modo, observa-se que foram encontrados valores acima do limite de segurança para consumo.

Lacerda et al. (2016) avaliaram as concentrações de Hg no músculo em S. cavalla (352 $\pm 468 \mathrm{ng} / \mathrm{g}^{-1}$ ) 
e S. brasiliensis $\left(126 \pm 108 \mathrm{ng} / \mathrm{g}^{-1}\right)$, obtendo valores médios menores em relação ao estudo de Costa et al. (2009a). Por ser a parte do pescado predominantemente consumida, o músculo pode apresentar um potencial risco de contaminação ao ser humano. Apesar dos valores médios de mercúrio encontrados nessas espécies o estudo revela que o nível de $\mathrm{Hg}$ observado em Cavala chegou a $1737 \mathrm{ng} / \mathrm{g}^{-1}$, ultrapassando o limite previsto na legislação.

Também relacionado ao fato de quanto mais elevado o nível trófico maior será a concentração de Hg acumulado, o trabalho de Milhomem Filho et al. (2016) mostrou maior concentração de Hg na espécie de peixe de hábito piscívoro $\left(0,2775 \pm 0,0551 \mu \mathrm{g} / \mathrm{g}^{-1}\right)$ enquanto o menor nível de $\mathrm{Hg}$ foi encontrado na espécie de hábito detritívoro $\left(0,0308 \pm 0,0108 \mu \mathrm{g} / \mathrm{g}^{-1}\right)$.

A concentração de mercúrio observada nas espécies de hábito herbívoro e/ou onívoro e zooplanctófago foi de 0,0506 $\pm 0,0183 \mu \mathrm{g} / \mathrm{g}^{-1}$ e 0,1360 $\pm 0,0985 \mu \mathrm{g} / \mathrm{g}^{-1}$, respectivamente. Nesse estudo, as concentrações estão abaixo do limite de segurança para consumo humano estabelecido.

\section{Concentração de Mercúrio em relação ao comprimento e peso}

Além da posição na cadeia trófica, a ocorrência deste metal no pescado pode ter influência de vários fatores, como a localização geográfica, e principalmente, o comprimento e peso do pescado por esse fator está associado à idade do peixe. O Hg se acumula ao longo do tempo e quanto mais jovem o peixe, menor o tempo de exposição ao mercúrio ${ }^{21}$. Em quatro estudos incluídos nesta revisão verificou-se a concentração de Hg em peixes e sua relação com o comprimento e o peso (COSTA et al., 2009a; LACERDA et al., 2016; COSTA et al., 2009b; COSTA et al., 2014).

No estudo de Costa et al. (2009a) valores acima do limite considerado seguro para consumo foram observados em indivíduos de S. cavalla de comprimento maior que $100 \mathrm{~cm}$. Lacerda et al. (2016) afirmaram que espécies piscívoras grandes podem mostrar concentrações de Hg maiores do que os limites aceitáveis para o consumo humano.

Ao avaliar outras 16 espécies Lacerda et al. (2016) mostraram que espécies de comprimento maior apresentam as concentrações de $\mathrm{Hg}$ mais elevadas, $509 \mathrm{ng} / \mathrm{g}^{-1} \mathrm{em} R$. porosus (Cação-frango) de $85 \mathrm{~cm}, 500 \pm$ 330 ng/g $/ \mathrm{g}^{-1}$ em G. cirratum (Tubarão-Lixa) de $185 \mathrm{~cm}$ e $231 \pm 43$ ng/g-1 em T. obesus (Atum) de $82 \pm 4,4 \mathrm{~cm}$. Níveis mais baixos de $\mathrm{Hg}$ foram encontrados em espécies de menor comprimento, $14 \pm 1,2 \mathrm{ng} / \mathrm{g}^{-1} \mathrm{em} S$. micrurum (Linguado) de 32,5 cm, $15 \pm 8,0 \mathrm{ng} / \mathrm{g}^{-1} \mathrm{em} H$. plumieri (Biquara) de 27,1 cm e $39 \pm 7,6 \mathrm{ng} / \mathrm{g}^{-1} \mathrm{em} P$. corvinaeformis (Coró) de 23,9 cm.

Da mesma forma, Costa et al. (2009b) ao avaliar a concentração de Hg no músculo da espécie Trichiurus lepturus (Peixe espada) relacionou o nível de $\mathrm{Hg}$ ao peso e comprimento do peixe. A concentração média de $\mathrm{Hg}$ encontrada foi de $125,3 \mu \mathrm{g} / \mathrm{kg}^{-1}\left(125,3 \mathrm{ng} / \mathrm{g}^{-1}\right)$, em indivíduos com média de 204,1g $\pm 97,9$ e $63,1 \mathrm{~cm} \pm 10,1$. Estes autores consideraram um intervalo para classificar indivíduos sub-adultos os que apresentaram de 30 a $70 \mathrm{~cm}$ e adultos os indivíduos com mais de $70 \mathrm{~cm}$. Apesar de encontrarem uma correlação positiva entre o teor de $\mathrm{Hg}$ e as variáveis de peso e comprimento total, o nível de Hg está abaixo do limite de segurança estabelecido. 
Valores bem menores $\left(0,051 \pm 0,031 \mu \mathrm{g} / \mathrm{g}^{-1}\right)$ foram encontrados em estudo semelhante com a mesma espécie (T. lepturus) realizado no Rio de Janeiro. De acordo com os autores Cardoso et al. (2009) o peixe sendo mais jovem considera-se que foi menos exposto ao $\mathrm{Hg}$, desse modo com menor risco de contaminação.

$\mathrm{O}$ valor de $\mathrm{Hg}$ pode ser atribuído ao fato da acumulação no organismo se dar com o tempo, e a diferença entre os tamanhos dos peixes pode influenciar na comparação de resultados.

Em seu estudo Costa et al. (2014) observaram a concentração de Hg em 13 espécies de peixe (com hábitos carnívoro, onívoro e detritívoro) e foi identificada correlação entre a concentração de $\mathrm{Hg}$ e dados de peso e comprimento em cinco dessas espécies. L. friderici (Piau) com média de $26,1 \pm 16,1 \mathrm{ng} / \mathrm{g}^{-1}, \mathrm{~S}$. rhombeus (Piranha-preta) 40,9 $\pm 24,8 \mathrm{ng} / \mathrm{g}^{-1}$, E. brasilianus (Carapeba) $21,3 \pm 19,2 \mathrm{ng} / \mathrm{g}^{-1}$, A. rhomboidalis (Salema) $49,2 \pm 27,4 \mathrm{ng} / \mathrm{g}^{-1}$ e $\mathrm{H}$. adscensionis (João-cachaça) $31,9 \pm 4,6 \mathrm{ng} / \mathrm{g}^{-1}$. Nenhuma das espécies estudadas nessa pesquisa apresentou concentração de $\mathrm{Hg}$ acima do limite de segurança.

\section{Acúmulo de mercúrio em peixes por meio de alimentação contaminada}

A contaminação dos peixes ocorre através de suas membranas por meio da absorção de mercúrio presente na água, outra forma de contaminação é por meio da alimentação, a quantidade de acúmulo depende da quantidade de alimento contaminado que foi ingerido (ISHIKAWA, 2003).

Um estudo apresentado nesta revisão verificou a presença de mercúrio na ração utilizada para alimentação de peixes em um reservatório da região Nordeste do Brasil e relacionou com a concentração de $\mathrm{Hg}$ em diversos órgãos. Oliveira et al. (2015) avaliaram a quantidade de $\mathrm{Hg}$ presente em cinco tipos de ração utilizada para alimentar peixe do tipo $O$. niloticus (Tilápia) encontrando maior concentração no tipo frita $\left(30,1 \pm 6,3 \mathrm{ng} / \mathrm{g}^{-1}\right)$ e menor concentração na alimentação oferecida na fase de engorda $\left(1,4 \pm 0,5 \mathrm{ng} / \mathrm{g}^{-1}\right)$, observaram o nível de Hg presente em diferentes órgãos deste peixe, as concentrações médias encontradas no músculo $\left(1,4 \pm 0,1 \mathrm{ng} / \mathrm{g}^{-1}\right)$, na pele $\left(1,1 \pm 0,1 \mathrm{ng} / \mathrm{g}^{-1}\right)$ e ossos $\left(1,0 \pm 0,7 \mathrm{ng} / \mathrm{g}^{-1}\right)$ foram menores em comparação com órgãos internos como fígado $\left(2,9 \pm 0,5 \mathrm{ng} / \mathrm{g}^{-1}\right)$ e coração $\left(2,5 \pm 0,1 \mathrm{ng} / \mathrm{g}^{-1}\right)$.

\section{Implicações de mercúrio na saúde}

Em humanos, a principal via de intoxicação por mercúrio é através do consumo de peixes contaminados, este alimento, então, é caracterizado habitualmente como o alimento consumido por humanos que mais concentra mercúrio (KITAHARA et al., 2000).

O mercúrio elementar $\left(\mathrm{Hg}^{\circ}\right)$ e o metil mercúrio $(\mathrm{MeHg})$ quando é absorvido, se acumula nos rins, no fígado e no sistema nervoso central, assim, a contaminação por mercúrio pode implicar em graves consequências a esses sistemas pois atua na inibição de enzimas e favorecem a inativação de proteínas (SILVEIRA et al., 2004; COSTA et al., 2014; MORGANO et al., 2005).

O Hg em excesso pode ocasionar transtornos neurológicos e comportamentais. Geralmente as manifestações clínicas podem ser agudas ou crônicas, os sintomas da intoxicação aguda são associados a tremores, insônia e efeitos neuromusculares. A constante exposição à baixas concentrações podem levar à intoxicação crônica ocasionando déficit cognitivo e motor, perda de visão e audição. Os sinais clínicos não 
ocorrem de imediato à exposição ao $\mathrm{Hg}$. As crianças são mais vulneráveis à contaminação por $\mathrm{Hg}$, podendo os sintomas ser ainda mais graves. Os sintomas neurológicos são retardo mental, convulsões e atraso no desenvolvimento (KASPER et al., 2007; WHO, 1990). Em gestantes, o excesso de consumo de peixes contaminados por $\mathrm{Hg}$ acima dos limites de segurança pode dificultar o desenvolvimento neurológico do feto (SILVEIRA et al., 2004; WHO, 1990).

Milhomem Filho et al. (2016) avaliaram as concentrações de mercúrio em famílias de pescadores no Maranhão. Ao avaliar amostras de cabelo observaram níveis médios de $\mathrm{Hg}$ no grupo masculino de 1,01 \pm $1,97 \mu \mathrm{g} / \mathrm{g}^{-1}$, e no feminino de $0,69 \pm 0,82 \mu \mathrm{g} / \mathrm{g}^{-1}$, considerando-se níveis aceitáveis de exposição, não apresentando risco. Esse mesmo estudo encontrou teores de $\mathrm{Hg}$ abaixo do limite de segurança nas espécies de peixes mais consumidas por essas famílias.

Algumas das espécies de peixe avaliadas nos estudos incluídos nesta revisão apresentaram níveis elevados de $\mathrm{Hg}$, estando próximo ou acima do limite seguro para consumo como o Cação-frango, o Tubarãolixa e Cavala (R. porosus, G. cirratum e S. cavalla). Portanto, ressalta-se que a inclusão dessas espécies no hábito alimentar deve ser moderada devido ao risco para a saúde.

\section{CONCLUSÕES}

Conclui-se que a maioria dos estudos apresentou concentrações totais de mercúrio abaixo do limite considerado seguro para consumo estabelecido pelas normas brasileiras. Porém, as espécies de peixes de maior porte e de hábitos carnívoros e piscívoros podem apresentar níveis de mercúrio acima desse limite. Portanto, deve-se observar com cautela a ingestão dietética desses tipos de pescado na região Nordeste. Observa-se a necessidade de mais estudos nessa região do país que avaliem a concentração de $\mathrm{Hg}$ e os efeitos do consumo de pescado na saúde dos seres humanos.

\section{REFERÊNCIAS}

ANVISA. Agência Nacional de Vigilância Sanitária. Resolução RDC n.42, 29 de agosto de 2013. Dispõe sobre o

Regulamento Técnico MERCOSUL sobre Limites Máximos de Contaminantes Inorgânicos em Alimentos. Brasília: ANVISA, 2013.

BRABO, E. S.; SANTOS, E. O.; JESUS, I. M.; MASCARENHAS, A. F.; FAIAL, K. F.. Níveis de mercúrio em peixes consumidos pela comunidade indígena de Sai Cinza na Reserva Munduruku, Município de Jacareacanga, Estado do Pará, Brasil. Cadernos de Saúde Pública, Rio de Janeiro, v.15, n.2, p.325-332, 1999. DOI: https://doi.org/10.1590/S0102$\underline{311 \times 1999000200017}$

BRASIL. Ministério do Meio Ambiente. Perfil do Gerenciamento de Mercúrio no Brasil, incluindo seus Resíduos. Brasília: MMA, 2013.

BURGER, J.; DIAZ-BARRIGA F.; MARAFANTE E.; POUNDS J.; ROBSON, M.. Methodologies to examine the importance of host factors in bioavailability of metals. Ecotoxicology Environmental Safety, v.56, n.1, p.20-31, 2003. DOI: https://doi.org/10.1016/S0147-6513(03)00047-2
CARDOSO, T. P.; MÁRSICO, E. T.; MEDEIROS, R. J.; TORTELLY, R.; SOBREIRO, L. G.. Concentração de mercúrio e análise histopatológica em músculo, rim e cérebro de peixe-espada (Trichiurus lepturus) coletados na praia de Itaipu, Niterói, Rio de Janeiro, Brasil. Ciência Rural, Santa Maria, v.39, n.2, p.540-546, 2009. DOI: https://doi.org/10.1590/S010384782008005000062

COSTA, B. G.; LACERDA, L. D.. Concentração de mercúrio total em Cavala, Scomberomorus cavalla, e Serra, Scomberomorus brasiliensis, comercializadas nas bancas de pescado do Mucuripe, Fortaleza, Ceará. Arquivos de Ciências do Mar, Fortaleza, v.42, n.1, p.22-29, 2009a. DOI: https://doi.org/10.32360/acmar.v42i1.6035

COSTA, M. F.; BARBOSA, S. C.; BARLETTA, M.; DANTAS, D. V.; KEHRING, H. A.; SEIXAS, T. G.; MALM, O.. Seasonal differences in mercury accumulation in Trichiurus lepturus (Cutlassfish) in relation to length and weight in a Northeast Brazilian estuary. Environmental Science and Pollution Research International, v.16, n.4, p.423-430, 2009b. DOI: https://doi.org/10.1007/s11356-009-0120-x 
COSTA, B. G.; LACERDA, L. D.. Mercury (Hg) in fish consumed by the local population of the Jaguaribe River lower basin, Northeast Brazil. Environmental Science and Pollution Research International, v.21, n.23, p.13335-13341, 2014. DOI: https://doi.org/10.1007/s11356-014-3297-6

KASPER, D.; BOTARO, D.; PALERMO, E. F. A.; MALM, O.. Mercúrio em peixes: fontes e contaminação. Oecologia Brasiliensis, Rio de Janeiro, v.11, n.2, p.228-239, 2007. DOI: https://doi.org/10.4257/oeco.2007.1102.07

KITAHARA, S. E.; OKADA, I. A.; SAMIKA, A. M.; ZENEBON, O.; JESUS, R. S.; TENUTA-FILHO, A.. Mercúrio total em pescado de água doce. Ciência e Tecnologia de Alimentos, Campinas, v.20, n.2, p.267-273, 2000. DOI:

https://doi.org/10.1590/S0101-20612000000200024

ISHIKAWA, N. M.. Toxicidade aguda e crônica do mercúrio em Tilápia Tailandesa, Oreochromis niloticus: determinação da CL50-96h e alterações hematológicas. Dissertação (Mestrado em Aquicultura) - Universidade Estadual Paulista, São Paulo, 2003.

LANDIS, J. R.; KOCH, G. G.. The measurement of observer agreement for categorical data. International Biometric Society, v.33, n.1, p.159-174, 1977. DOI: https://doi.org/10.2307/2529310

LACERDA, L. D.; BEZERRA, M. F.; COSTA, B. G. B.; BRAGA, T. M.; GOYANNA, F. A. A.. Mercury distribution in fish commercialized at the mucuripe market, Fortaleza, Ceará state, Brazil. Arquivos de Ciências do Mar, Fortaleza, v.49, n.1, p.50-54, 2016. DOI:

https://doi.org/10.32360/acmar.v49i1.6159

LACERDA, L. D.; MALM, O.. Contaminação por mercúrio em ecossistemas aquáticos: uma análise das áreas críticas. Estudos Avançados, São Paulo, v.22, n.63, p.173190, 2008. DOI: https://doi.org/10.1590/S0103$\underline{40142008000200011}$

LACERDA, L. D.; SANTOS, J. A.; CAMPOS, R. C.; GONÇALVES, R. A.; SALLES, R.. Total-Hg and organic-Hg in Cephalopholis fulva (Linnaeus, 1758) from inshore and offshore waters of NE Brazil. Brazilian Journal of Biology, São Paulo, v.7, n.3, p.493-498, 2007. DOI: https://doi.org/10.1590/S1519$\underline{69842007000300014}$

MENDES, K. S.; SILVEIRA, R. C. C. P.; GALVÃO, C. M.. Revisão integrativa: método de pesquisa para a incorporação de evidências na saúde e na enfermagem. Texto \& Contexto -
Enfermagem, Florianópolis, v.17, n.4, p.758-764, 2008. DOI: https://doi.org/10.1590/S0104-07072008000400018

MICARONI, R. C. C. M.; BUENO, M. I. M. S.; JARDIM, W. F.. Compostos de mercúrio: Revisão de métodos de determinação, tratamento e descarte. Química Nova, v.23, n.4, p.487-495, 2000. DOI: https://doi.org/10.1590/S0100$\underline{40422000000400011}$

MILHOMEM FILHO, E. O.; OLIVEIRA, C. S. B.; SILVEIRA, L. C. L.; CRUZ, T. M.; SOUZA, G. S.; COSTA JUNIOR, J. M. F.; PINHEIRO, M. C. N.. A ingestão de pescado e as concentrações de mercúrio em famílias de pescadores de Imperatriz (MA). Revista Brasileira de Epidemiologia, v.19, n.1, p.14-25, 2016. DOI: https://doi.org/10.1590/1980$\underline{5497201600010002}$

MORGANO, M. A.; GOMES, P. C.; MANTOVANI, D. M. B.; PERRONE, A. A. M.; SANTOS, T. F.. Níveis de mercúrio total em peixes de água doce de pisciculturas paulistas. Ciências e Tecnologia de Alimentos, Campinas, v.25, n.2, p.250-253, 2005.

OLIVEIRA, J. F.; NOVAES, J. L. C.; MORAES SEGUNDO, A. L. N.; PERETTI, D.. Caracterização da pesca e percepção de pescadores artesanais em uma Reserva de Desenvolvimento Sustentável no Nordeste brasileiro. Natureza, v.14, n.1, p.48-54, 2016.

OLIVEIRA, K. F.; LACERDA, L. D.; PERES, T. F.; BEZERRA, M. F.; DIAS, F. J.. Emission factor and balance of mercury in fish farms in an artificial reservoir in NE Brazil. Environmental science and pollution research international, v.22, n.22, p.18278-18287, 2015. DOI: https://doi.org/10.1007/s11356$\underline{015-5102-6}$

SILVEIRA, L. C. L.; VENTURA, D. F.; PINHEIRO, M. C. N.. Toxicidade mercurial: avaliação do sistema visual em indivíduos expostos a níveis tóxicos de mercúrio. Ciências e Cultura, São Paulo, v.56, p.36-38, 2004.

TINÔCO, A. A. P.; AZEVEDO, I. C. A. D.; MARQUES, E. A. G.; MOUNTEER, A. H.; MARTINS, C. P.; NASCENTES, R.; REIS, E. L.; NATALINO, R.. Avaliação de contaminação por mercúrio em Descoberto, MG. Engenharia Sanitária Ambiental, Rio de Janeiro, v.15, n.4, p.305-314, 2010. DOI: https://doi.org/10.1590/S1413-41522010000400003

WHO. World Health Organization. Environmental Health Criteria 101: Methylmercury. Geneva: International Programme On Chemical Safety, 1990.

A CBPC - Companhia Brasileira de Produção Científica (CNPJ: 11.221.422/0001-03) detém os direitos materiais desta publicação. Os direitos referem-se à publicação do trabalho em qualquer parte do mundo, incluindo os direitos às renovações, expansões e disseminações da contribuição, bem como outros direitos subsidiários. Todos os trabalhos publicados eletronicamente poderão posteriormente ser publicados em coletâneas impressas sob coordenação da Sustenere Publishing, da Companhia Brasileira de Produção Científica e seus parceiros autorizados. Os (as) autores (as) preservam os direitos autorais, mas não têm permissão para a publicação da contribuição em outro meio, impresso ou digital, em português ou em tradução. 